\title{
Graphene oxide selectively targets cancer stem cells, across multiple tumor types: Implications for non-toxic cancer treatment, via "differentiation-based nano-therapy"
}

\author{
Marco Fiorillo ${ }^{1,2,3}$, Andrea F. Verre ${ }^{4}$, Maria Iliut ${ }^{4}$, Maria Peiris-Pagés ${ }^{1,2}$, Bela \\ Ozsvari,1,2, Ricardo Gandara ${ }^{1,2}$, Anna Rita Cappello3, Federica Sotgia1,2, Aravind \\ Vijayaraghavan ${ }^{4}$ and Michael P. Lisanti, ${ }^{1,2}$ \\ ${ }^{1}$ The Manchester Centre for Cellular Metabolism (MCCM), Institute of Cancer Sciences, University of Manchester, UK \\ 2 The Breakthrough Breast Cancer Research Unit, Institute of Cancer Sciences, University of Manchester, UK \\ ${ }^{3}$ The Department of Pharmacy, Health and Nutritional Sciences, The University of Calabria, Italy \\ ${ }^{4}$ School of Materials and National Graphene Institute, University of Manchester, UK \\ Correspondence to: Michael P. Lisanti, email: michael.lisanti@manchester.ac.uk
}

Aravind Vijayaraghavan, email: aravind@manchester.ac.uk

Keywords: nanomaterials, graphene oxide, cancer stem cells, multiple cancer types: breast, ovarian Received: January 01, $2015 \quad$ Accepted: February 12, $2015 \quad$ Published: February 24, 2015

This is an open-access article distributed under the terms of the Creative Commons Attribution License, which permits unrestricted use, distribution, and reproduction in any medium, provided the original author and source are credited.

\section{ABSTRACT}

Tumor-initiating cells (TICs), a.k.a. cancer stem cells (CSCs), are difficult to eradicate with conventional approaches to cancer treatment, such as chemo-therapy and radiation. As a consequence, the survival of residual CSCs is thought to drive the onset of tumor recurrence, distant metastasis, and drug-resistance, which is a significant clinical problem for the effective treatment of cancer. Thus, novel approaches to cancer therapy are needed urgently, to address this clinical need. Towards this end, here we have investigated the therapeutic potential of graphene oxide to target cancer stem cells. Graphene and its derivatives are well-known, relatively inert and potentially non-toxic nano-materials that form stable dispersions in a variety of solvents. Here, we show that graphene oxide (of both big and small flake sizes) can be used to selectively inhibit the proliferative expansion of cancer stem cells, across multiple tumor types. For this purpose, we employed the tumorsphere assay, which functionally measures the clonal expansion of single cancer stem cells under anchorage-independent conditions. More specifically, we show that graphene oxide effectively inhibits tumor-sphere formation in multiple cell lines, across 6 different cancer types, including breast, ovarian, prostate, lung and pancreatic cancers, as well as glioblastoma (brain). In striking contrast, graphene oxide is nontoxic for "bulk" cancer cells (non-stem) and normal fibroblasts. Mechanistically, we present evidence that GO exerts its striking effects on CSCs by inhibiting several key signal transduction pathways (WNT, Notch and STAT-signaling) and thereby inducing CSC differentiation. Thus, graphene oxide may be an effective non-toxic therapeutic strategy for the eradication of cancer stem cells, via differentiation-based nanotherapy.

\section{INTRODUCTION}

Cancer stem cells (CSCs) are resistant to conventional therapeutic approaches [1-3]. As a consequence, they have been directly implicated in the disease pathogenesis of tumor recurrence and distant metastasis [4, 5]. In addition, drug-resistant CSCs have been linked to unfavorable clinical outcomes, 
across different tumor types [6-8]. As only a very small percentage of cancer cells have "stem-like" and "tumor-initiating" properties, they are difficult to study and their key distinguishing features remain relatively uncharacterized, although they appears to resist both chemo-therapy and radiation.

Interestingly, CSCs share many properties with normal stem cells, including immortality and resistance to stress, as well as asymmetric cell division [9, 10]. A particular distinguishing characteristic of CSCs is their ability to initiate tumors and to undergo anchorageindependent growth, when cultured in suspension [11]. Under these particular cell culture conditions, CSCs proliferate and form 3D-spheroid-like structures, containing CSCs and progenitor cells, which are known as "tumor-spheres" or "onco-spheres" [12, 13]. In striking contrast, the vast majority of non-CSCs undergo a specialized form of apoptosis in suspension cultures, called anoikis. Importantly, each 3D-spheroid originates from the clonal proliferation of a single CSC, is not to due to the self-aggregation of cancer cells. Thus, tumor-sphere formation is an efficient means to selectively enrich for CSCs. The CSC population is resistant to DNA-damage, and shows lower levels of ROS production, as well [14, 15]. 3D-tumor-spheres derived from breast cancer cells are also known as mammo-spheres $[12,13]$.

Clinically, there is an urgent need to identify new therapeutic strategies for selectively targeting CSCs. Here, we show that graphene oxide (GO) demonstrates this selectivity. As such, our current study provides a new rationale for exploiting graphene oxide itself as an anticancer therapeutic, rather than simply as a drug-delivery agent [16].

\section{RESULTS}

\section{GO flakes target breast cancer stem cells}

Here, we tested the efficacy of graphene oxide as a potential new anti-cancer agent, for the selective targeting of CSCs. Graphene, described as two-dimensional sheets of carbon atoms without any additional functional groups, does not forms stable dispersions in water or other biologically relevant solvents [17]. On the other hand, graphene oxide [18] is the water-soluble derivative of graphene which can be produced with various sizes and bearing varied functional groups and which can be more easily manipulated experimentally, especially in biological systems. Sterile GO dispersions were prepared in a $5 \%$ mixture of DMSO in double-distilled water for these studies.

Initially, we tested the ability of GO to affect CSC proliferation, using MCF7 cells, a well-established ER $(+)$ breast cancer cell line. Two grades of GO were used, small GO (s-GO) with flake sizes of $0.2-2 \mu \mathrm{m}$ and big GO (b-GO) with flake sizes of 5-20 $\mu \mathrm{m}$, to represent two size classes, where the flakes are either smaller or larger than the target cells (Figure 1). For this purpose, we assessed the effects of graphene oxide on the anchorageindependent clonal expansion of MCF7 CSCs, using the tumor-sphere assay. This functional assay directly measures CSCs proliferative expansion [12]. Figure 2 shows the results of this analysis. Using s-GO flakes, we observed a dose-dependent inhibition of tumor-sphere formation, in the range of 1.25 to $25 \mu \mathrm{g} / \mathrm{ml}$, with an IC-

\section{Big GO}

\section{Small GO}

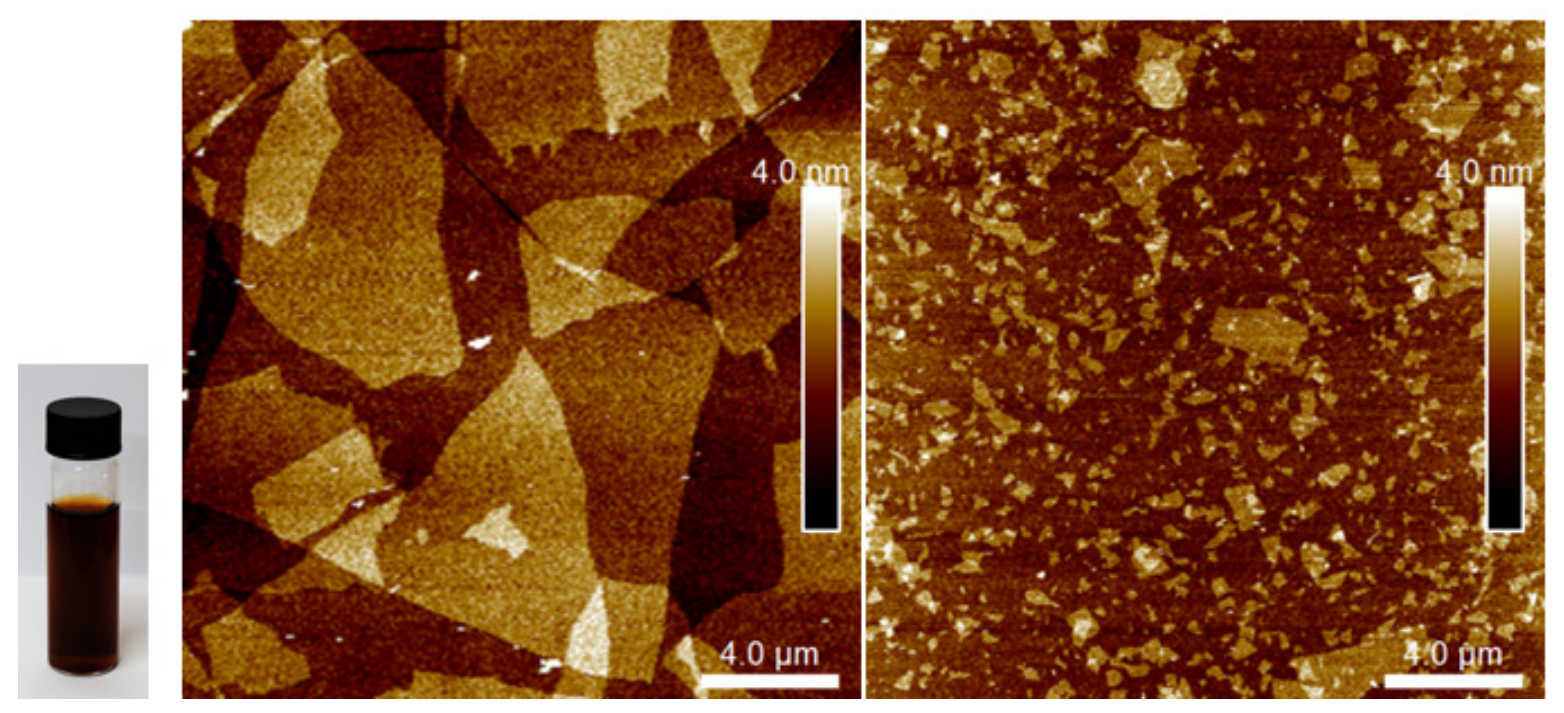

Figure 1: Graphene oxide (GO) grades. Left and Right panels show atomic force microscopy images of graphene oxide on a silicon dioxide substrate indicating the flake size distribution and monolayer thickness of the flakes. Inset shows a vial of b-GO stock dispersion in DMSO and water, at concentration of $2.3 \mathrm{mg} / \mathrm{ml}$. 
Small GO: MCF7 Cell Mammospheres
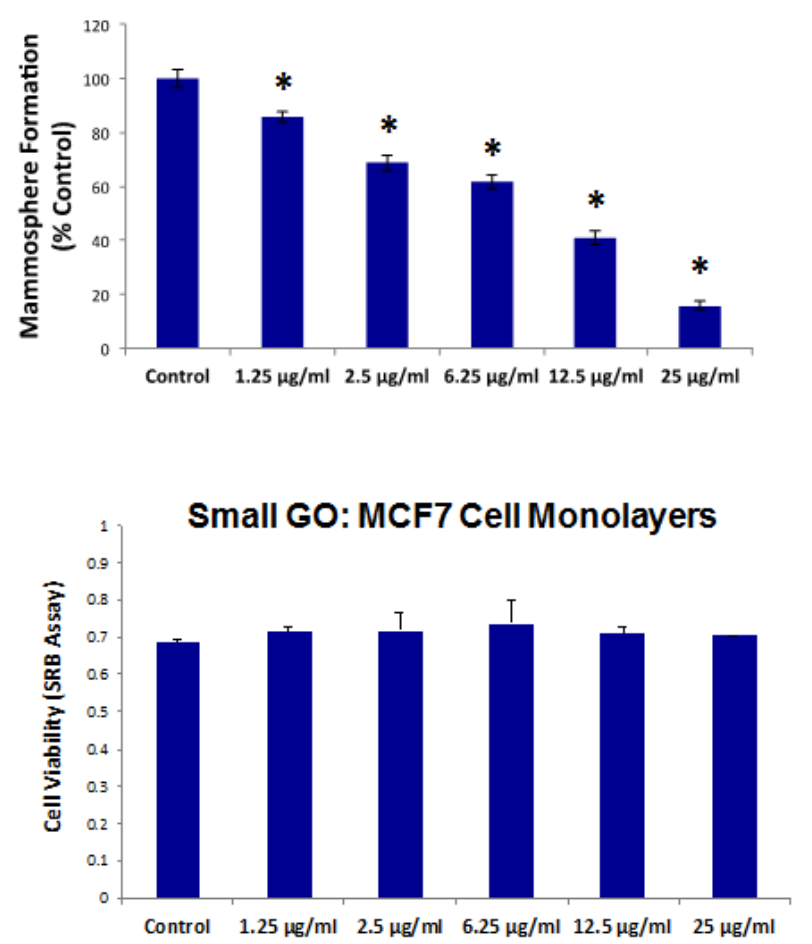

Big GO: MCF7 Cell Mammospheres

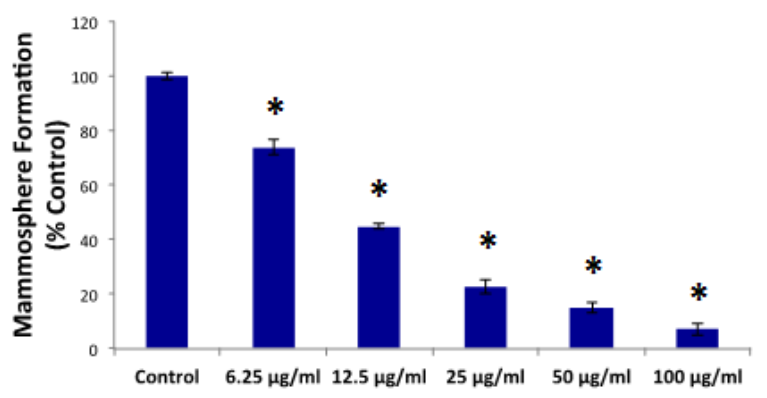

Big GO: MCF7 Cell Monolayers

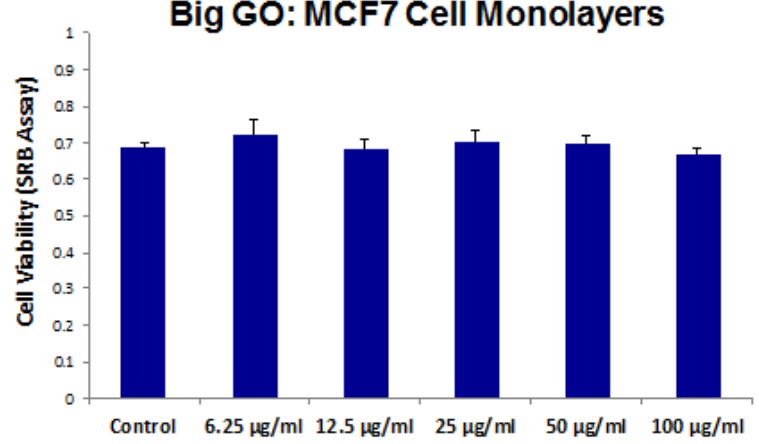

Figure 2: Graphene oxide (GO) selectively targets cancer stem cells (CSCs) in breast cancer cells. Upper Panels. Note that GO (big and small flakes) inhibits the anchorage-independent proliferation of MCF7 CSCs, as evidenced by inhibition of mammosphere formation. Lower Panels. In contrast, GO (big and small flakes) does not affect cell viability of the total MCF7 cell population. An * indicates $\mathrm{p}<0.05$ (Student's t-test).

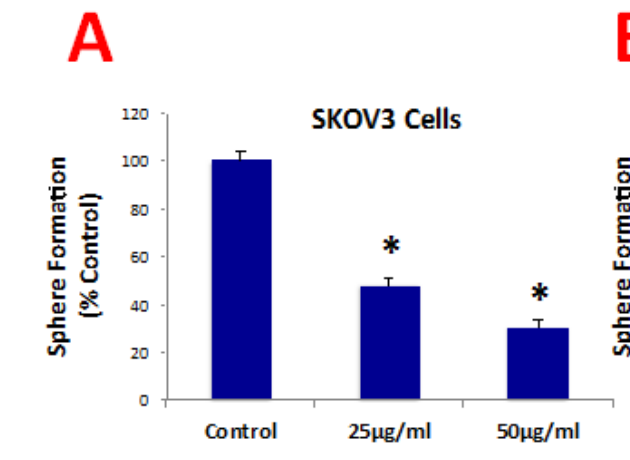

B
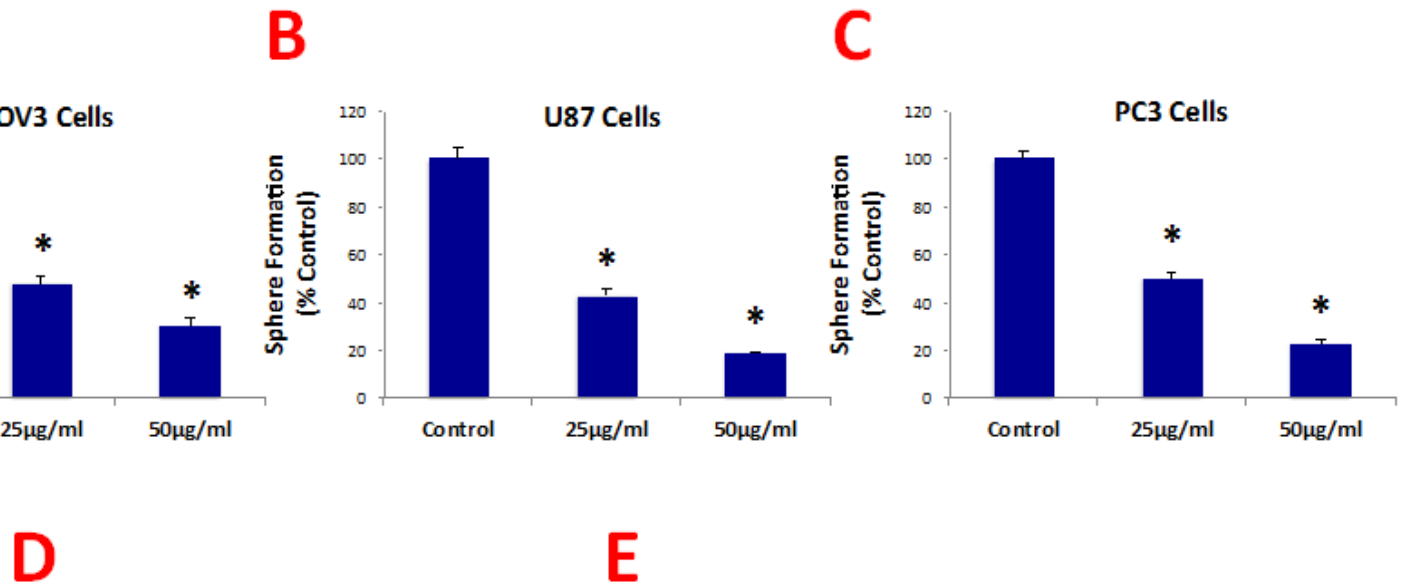

E
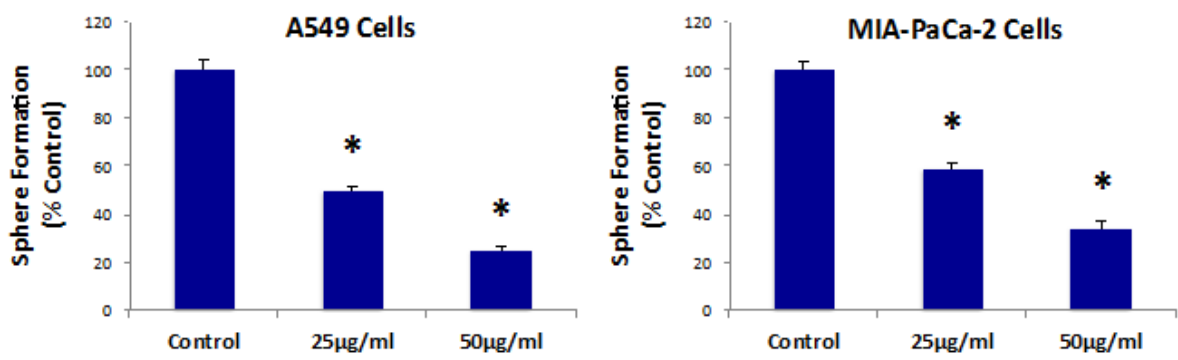

Figure 3: Graphene oxide (GO) selectively targets cancer stem cells (CSCs) of multiple cancer cell types. GO (big flakes) inhibits the anchorage-independent proliferation of SKOV3 ovarian cancer cells (A), U87 glioblastoma cells (B), PC3 prostate cancer cells (C), A549 lung cancer cells (D), as well as pancreatic cancer cells (E), in a concentration-independent manner. These results indicate that $\mathrm{GO}$ inhibits sphere formations of multiple cancer types. An * indicates $\mathrm{p}<0.05$ (Student's t-test). 
Table 1: Six Cancer Cell Models with Broad Applicability.

\begin{tabular}{l|l}
\hline Cancer Types & Cell Lines \\
\hline Breast(ER+) & MCF7 \\
\hline Ovarian & SKOV3 \\
\hline Prostate & PC3 \\
\hline Pancreatic & MIA-PaCa-2 \\
\hline Lung & A549 \\
\hline Glioblastoma & U87 MG
\end{tabular}

50 of $\sim 12.5 \mu \mathrm{g} / \mathrm{ml}$. Similarly, using b-GO flakes, we also observed a dose-dependent inhibition of tumor-sphere formation, in the range of 6.25 to $100 \mu \mathrm{g} / \mathrm{ml}$, again with an IC-50 of $\sim 12.5 \mu \mathrm{g} / \mathrm{ml}$. Importantly, both small and big GO flakes did not affect the viability of the bulk non-CSC population of MCF7 cells, indicating selectivity towards CSCs (Figure 2).

\section{GO flakes target CSCs, across multiple cancer types}

Since both the small and big GO flakes showed similar potency, we focused more on evaluating the efficacy of b-GO flakes. We next evaluated whether GO also showed efficacy against CSCs from multiple cancer types, such as ovarian, prostate, pancreatic and lung cancers, as well as glioblastoma (brain) (the six cell lines tested are summarized in Table 1). For simplicity, we tested b-GO flakes at two doses, namely 25 and $50 \mu \mathrm{g} / \mathrm{ml}$.

Figure 3 shows that b-GO flakes also effectively inhibited tumor-sphere formation in these 5 other cell lines. Thus, our results indicate that GO must be targeting a relatively specific and highly-conserved phenotypic property of CSCs, across multiple cancer types. Representative images of tumor-sphere inhibition by GO treatment are shown in Figure 4. Interestingly, the viability of bulk non-CSCs from these five cancer cell lines (SKOV3, PC3, A549, Mia PaCa2, and U-87) was not affected by GO (Figure 5), further highlighting its specificity and selectivity for CSCs.

Importantly, b-GO flakes also did not affect the viability of a normal skin fibroblast cell line (hTERT$\mathrm{BJ} 1$ ), indicating that GO is relatively non-toxic for normal body cells (Figure 6). This is consistent with the findings of many other laboratories, i.e., that GO is non-toxic for multiple normal cell types $[19,20]$.
GO-based mechanistic studies: Effects on wellestablished CSC signaling pathways

To gain mechanistic insights into how GO flakes target cancer stem cells, we next analyzed their effects on a series of well-established signal transduction pathways, which have been shown to contribute towards "stemness" [21-23].

For this purpose, we used a panel of MCF7 cell lines that were stably-transfected with different luciferase reporters, that allows one to quantitatively measure the activation-state of a given signal transduction pathway. Interestingly, Figure 7 shows that a number of signaling pathways were significantly inhibited by GO treatment. More specifically, GO treatment inhibited WNT- and Notch-driven signaling, as well as STAT1/3 signaling and the NRF2-dependent anti-oxidant response. However, little or no effect was observed on TGF-beta/SMAD-

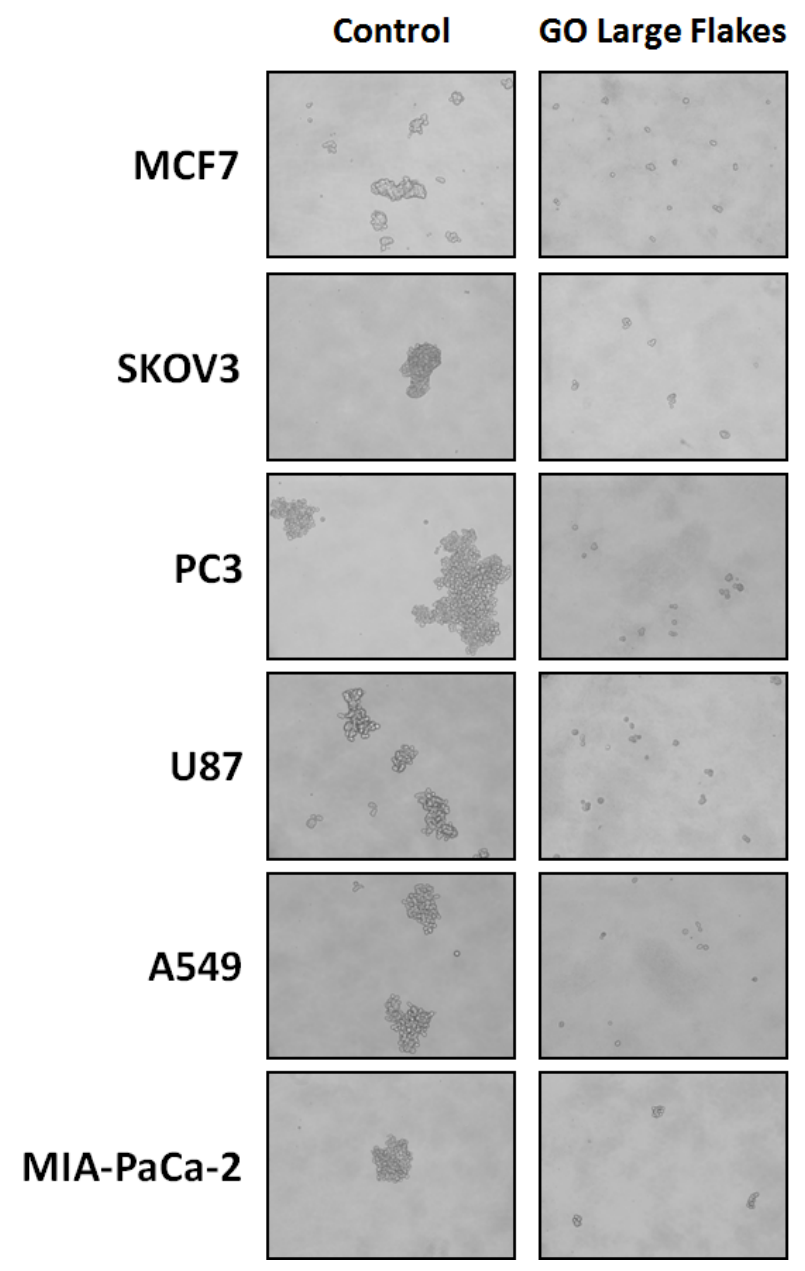

Figure 4: Graphene oxide (GO) selectively targets cancer stem cells (CSCs) of multiple cancer cell types. Representative images showing that GO (big flakes, $25 \mu \mathrm{g} / \mathrm{ml}$ ) inhibits the anchorage-independent proliferation of MCF7 breast cancer cells, SKOV3 ovarian cancer cells, PC3 prostate cancer cells, U87 glioblastoma cells, A549 lung cancer cells as well as MIA-PaCa-2 pancreatic cancer cells. 
signaling (Figure 7).

Thus, it appears that GO treatment somehow targets several different signal transduction pathways in cancer cells, to reduce overall "stemness".

\section{GO promotes the differentiation of breast cancer stem cells}

To further validate the idea GO was reducing stemness in MCF7-derived CSCs, we used a series of well-established breast cancer stem cell markers (CD44 and CD24), and quantitatively analyzed their expression by FACS analysis. The results of these studies are presented in Figure 8.

Briefly, MCF7 cells were treated as monolayer cultures with GO $(50 \mu \mathrm{g} / \mathrm{ml})$ for 48 hours or left untreated (vehicle alone control). Then, cells were trypsinized and plated on low-attachment plates for 10 hours to induce anoikis and enrich for CSCs. Single cells were then analysed by FACS to quantitate the CD44(+)CD24-/ low population, which represents the breast CSCs. As predicted, the CD44(+)CD24-/low population is greatly enriched after 10 hours in low-attachment conditions
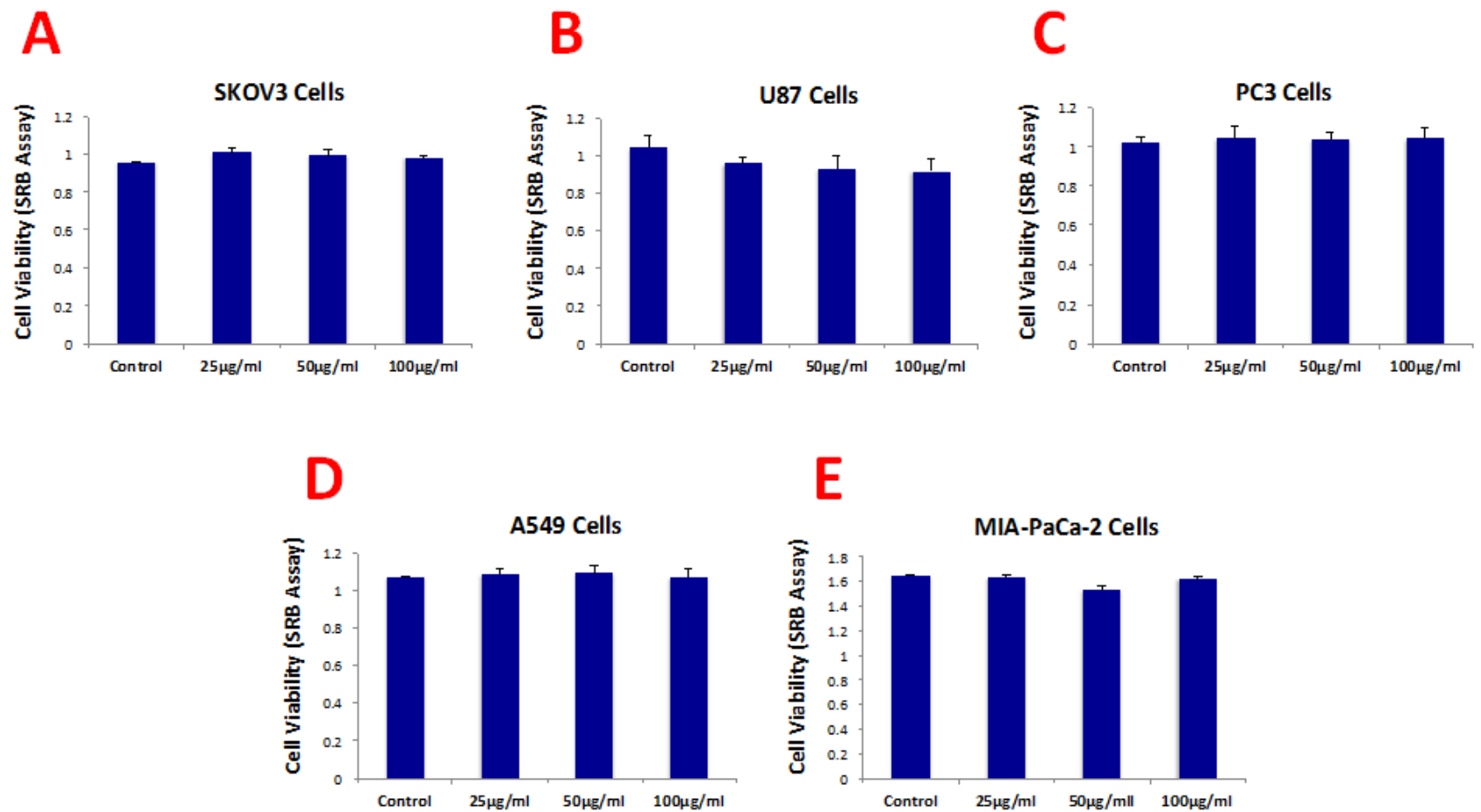

Figure 5: Graphene oxide (GO) does not affect cell viability of the total population of cancer cells. Cell viability was assessed using an SRB assay. Note that GO (big flakes) does not affect cell viability of the total cell population of SKOV3 ovarian cancer cells (A), U87 glioblastoma cells (B), PC3 prostate cancer cells (C), A540 lung cancer cells (D) as well as MIA-PaCa-2 pancreatic cancer cells.

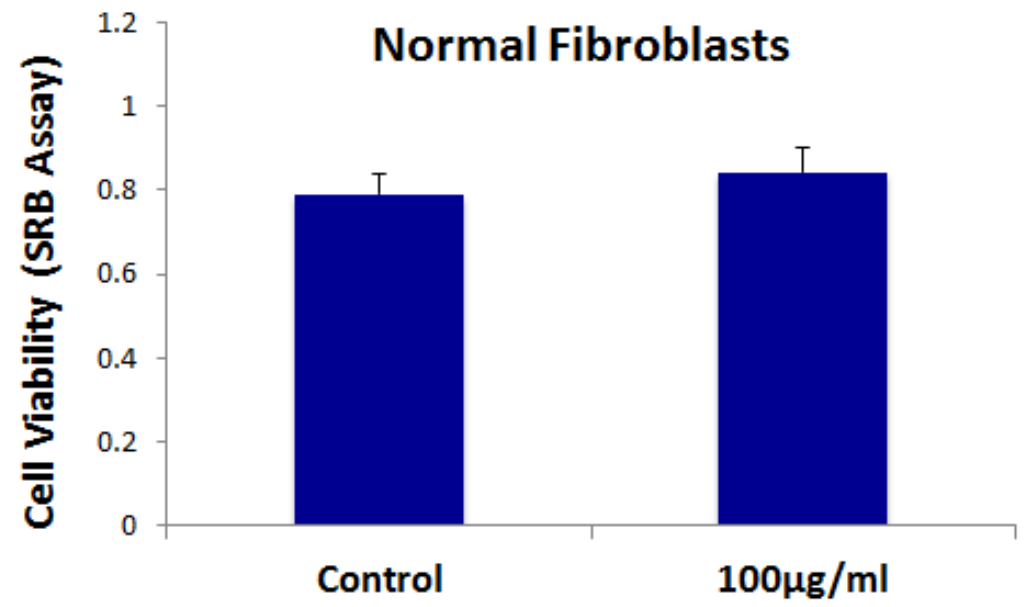

Figure 6: Graphene oxide (GO) does not affect the cell viability normal fibroblasts. Cell viability of hTERT-BJ1 fibroblasts was assessed using an SRB assay. Note that GO (big flakes) does not affect cell viability of the total cell population of normal fibroblasts. 

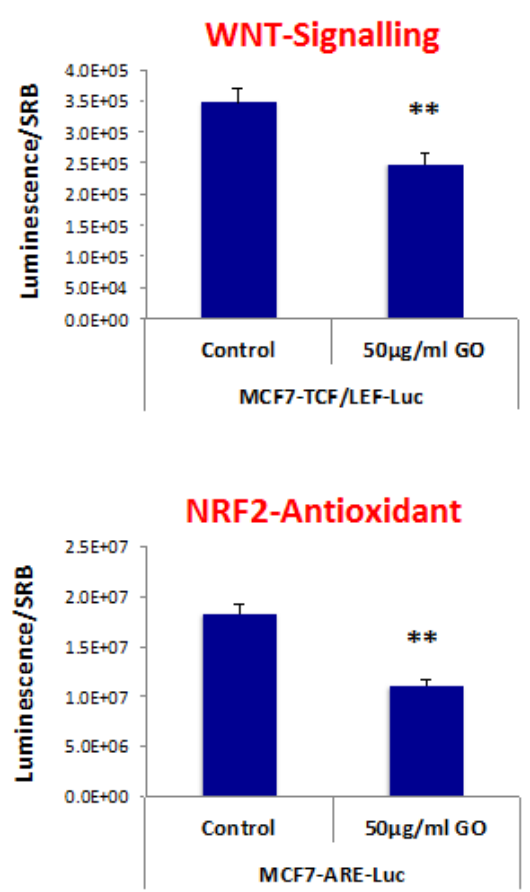

STAT3-Signalling

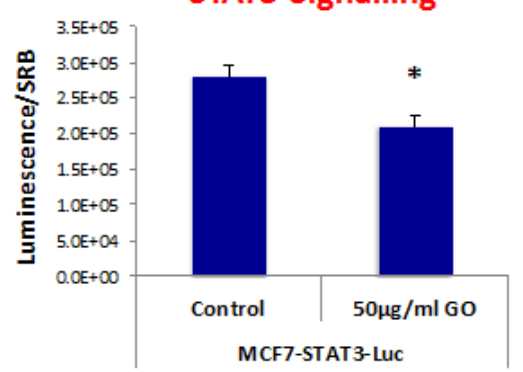

INFY-STAT1-Signalling

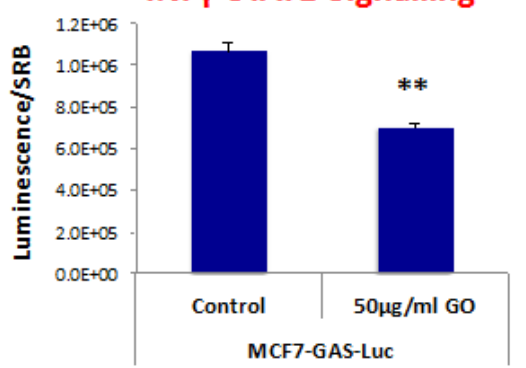

Notch-Signalling
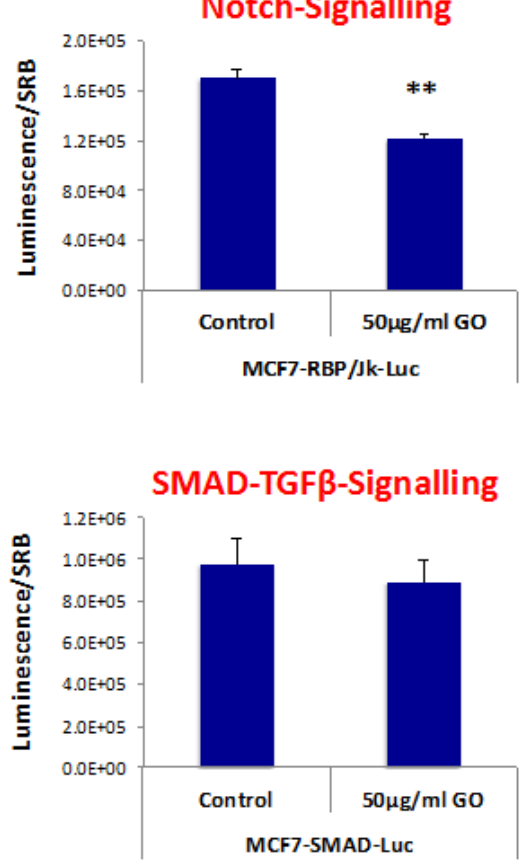

Figure 7: Graphene oxide (GO) inhibits signaling pathways related to cancer stem cells, antioxidant responses and interferon. MCF7 breast cancer cells carrying luciferase-reporters (Cignal, QIAGEN) were generated to monitor the activation of a variety of signaling networks, including Wnt, STAT3, Notch, NRF2-dependent antioxidant responses, Interferony-STAT1 and SMADTGF $\beta$ pathways. MCF7-Luc reporter cells were treated with GO (big flakes) for 48 hours and luminescence was determined as a measure of pathway activation status. Note that GO inhibits cancer stem cell signaling (WNT, STAT3 and Notch), NRF2-antioxidant responses, as well as INFY-STAT1 signaling. No effects were observed for the SMAD-TGF $\beta$-pathway. An * indicates $\mathrm{p}<0.05 ; * *$ indicates $\mathrm{p}<0.01$ (Student's t-test).
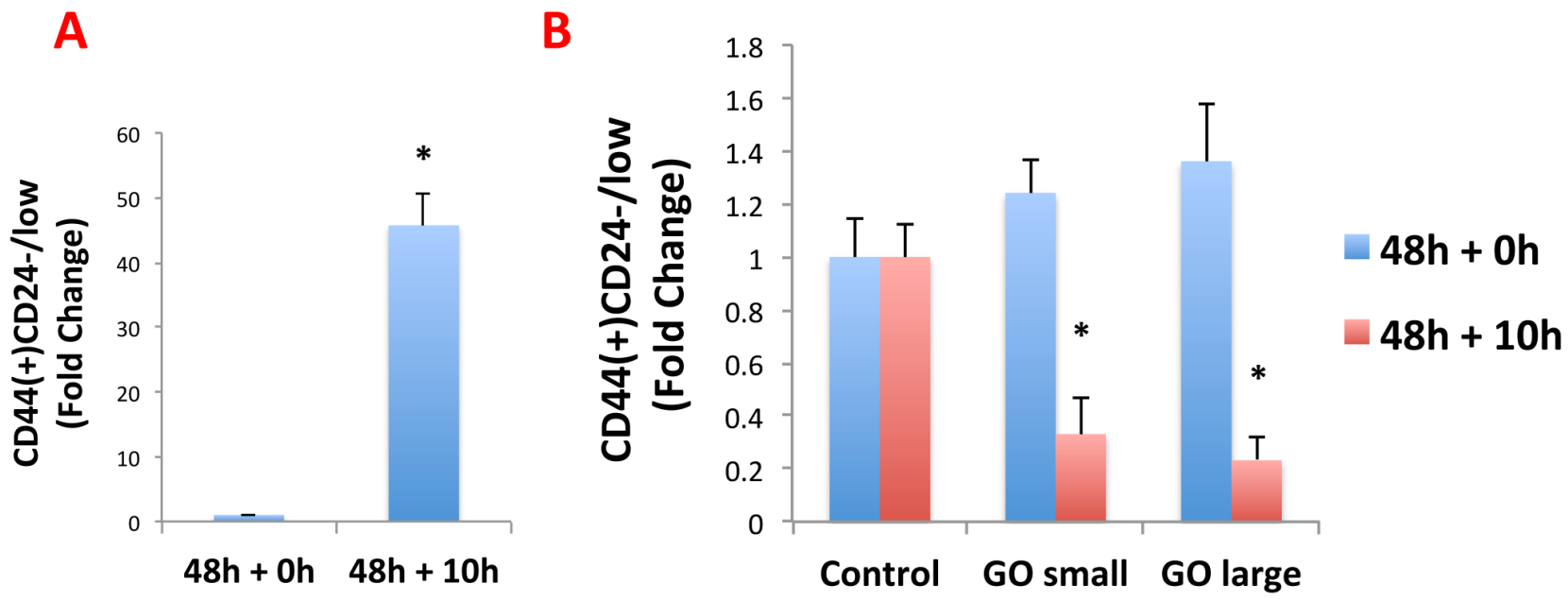

Figure 8: Graphene oxide (GO) promotes the differentiation of breast cancer stem cells. MCF7 cells were treated as monolayer cultures with small or big $\mathrm{GO}(50 \mu \mathrm{g} / \mathrm{ml})$ for 48 hours or left untreated (vehicle alone control). Then, cells were trypsinized and plated on low-attachment plates for 10 hours to induce anoikis and enrich for cancer stem cells. Single cells were then analyzed by FACS to quantitate the CD44(+)CD24-/low population, which represents the cancer stem cells. A. Note that, as expected, the CD44(+)CD24-/ low population is greatly enriched after 10 hours in low-attachment conditions (vehicle alone control). B. Interestingly, GO does not reduce the total number of anoikis-resistant cells (data not shown), but rather induces the expression of $\mathrm{CD} 24$, thereby significantly reducing the $\mathrm{CD} 44(+) \mathrm{CD} 24-/$ low population. This suggests that GO inhibits mammosphere formation by promoting the differentiation of breast cancer stem cells. An * indicates $\mathrm{p}<0.05$ (Student's t-test). 
(vehicle alone control) (Figure 8A).

Interestingly, $\mathrm{GO}$ does not reduce the total number of anoikis-resistant cells (data not shown), but rather induces the expression of $\mathrm{CD} 24$, thereby significantly reducing the $\mathrm{CD} 44(+) \mathrm{CD} 24-/$ low population (Figure 8B). This suggests that GO inhibits mammosphere formation by promoting the differentiation of breast cancer stem cells, supporting our results from the analysis of multiple signal transduction pathways

\section{DISCUSSION}

Here, we show that treatment with GO is sufficient to inhibit tumor-sphere formation in six independent cancer cell lines, across multiple tumor types (breast, ovarian, prostate, lung, and pancreatic cancer, as well glioblastoma (brain cancer)). These results suggest that GO specifically targets a global phenotypic property of CSCs that is highly conserved in multiple tumor types. Moreover, using MCF7 cells expressing a panel of luciferase reporters, we observed that GO treatment was indeed sufficient to inhibit a number of different signal transduction pathways, including WNT, Notch, STAT1/3 and NRF-2, but did not effect TGB-beta/SMAD signaling. Finally, using a panel of specific well-established breast CSC markers (namely CD44 and CD24), we show that GO appears to reduce the number of CSCs by inducing their differentiation, as they now begin to express CD24.
Thus, GO may reduce the number of bonafide CSCs that are capable of forming tumor-spheres, by inducing their differentiation and inhibiting their proliferation. However, additional mechanistic studies are clearly warranted.

Importantly, our preliminary results indicate that GO treatment does not significantly affect oxidative mitochondrial metabolism (OXPHOS) in this context (data not shown), suggesting that GO does not target mitochondria. This is in contrast to our previous studies where a number of mitochondrially-targeted FDAapproved antibiotics effectively eradiated CSCs [24]. Thus, GO and mitochondrially-targeted antibiotics appear to work differently, via separate and distinct molecular mechanism(s).

Also, since b-GO flakes are 5-to- $20 \mu \mathrm{m}$ in size, they must be exerting their effects at the cell surface, as they are too large to be internalized within cells and are actually larger than a single cell. This is consistent with our findings that GO-treatment dampens the activation of several stem cell associated signal transduction pathways, which are initiated at the cell surface. This could then mechanistically induce CSC differentiation, which we observed experimentally (summarized in Figure 9).

Previous studies have shown that GO (or its related derivatives) can inhibit "bulk" cancer cell migration or prevent tumor growth in xenograft models [25-28]. However, none of these studies connected GO treatment to the CSC phenotype or indicated that it could be used for "differentiation" therapy.

\section{Differentiation-Based Nano-Therapy}

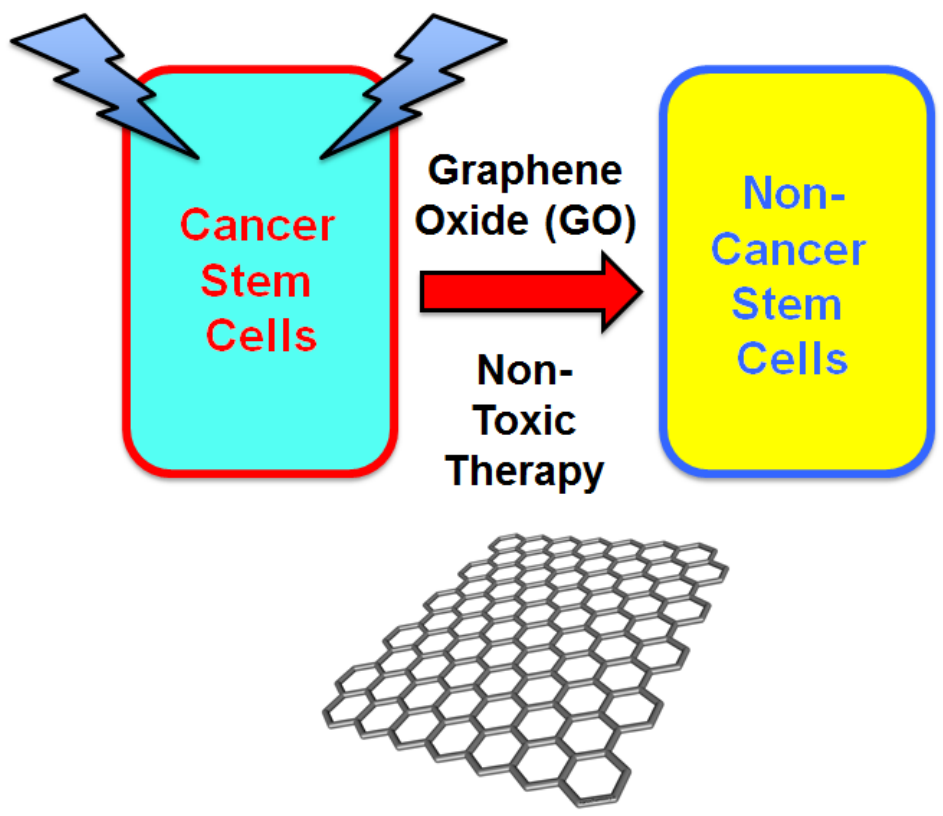

Figure 9: Graphene oxide (GO): Targeting cancer stem cells with differentiation-based nano-therapy. Our current mechanistic studies suggest that GO could directly be used as a therapeutic for targeting CSCs, because of its ability to induce differentiation. In this context, we might envision that GO could used to clear residual CSCs, with the aim of preventing tumor recurrence and distant metastasis, thereby providing a practical means for achieving "differentiation-based nano-therapy". 
Interestingly, several studies have shown that $\mathrm{GO}$ is non-toxic for normal stem cells, and indeed GO promotes their differentiation. More specifically, it was demonstrated that culturing normal pluripotent stem cells on GO as a substrate induces their terminal differentiation towards multiple cell lineages, including neurons, chondrocytes and adipocytes [29-33]. These properties are currently being actively exploited for tissue engineering and regenerative medicine, by using GO as a scaffold for bone reconstruction and neural regeneration.

Our new mechanistic studies suggest that GO could directly be used as a therapeutic for targeting CSCs, possibly as a differentiation agent. In this context, we envision that GO could be delivered i.v. or p.o., as a new anti-cancer therapeutic, depending on the location of the tumor. Alternatively, GO flakes could also be used as a lavage solution during surgery, to clear the tumor excision site or the peritoneal cavity (as in ovarian or other peritoneal cancers) of residual CSCs, with the aim of preventing tumor recurrence and distant metastasis, via differentiation-based nano-therapy (Figure 9).

\section{MATERIALS \& METHODS}

\section{Materials}

Cancer cell lines were purchased from the ATCC or other commercially available sources. Gibco-brand cell culture media (DMEM/F12) was purchased from Life Technologies.

\section{Graphene oxide}

Graphene oxide was prepared by using the Hummers method with modifications [34, 35]. The individual graphite oxide flakes contain carboxyl groups mainly at the edges, and epoxide, hydroxide and ketone groups mainly on the basal plane. The $\mathrm{C}$ to $\mathrm{O}$ ratio is usually slightly lower or slightly higher than 1 as determined by $\mathrm{X}$-ray photoemission spectroscopy. The graphene oxide flakes of different sizes were separated by centrifuging graphene oxide suspensions at various rpm and collecting different phases of the suspension. The AFM characterization of graphene oxide flakes was performed on a Bruker Dimension FastScan AFM system by using taping mode. The substrates were prepared by spin-casting the suspension on a $\mathrm{Si} / \mathrm{SiO} 2$ substrate to yield monolayer film, followed by AFM imaging. Concentrations were obtained from UV-Vis spectra, which were recorded in 10 $\mathrm{mm}$ path length quartz cells using a PerkinElmer Lambda - 1050 UV-Vis-NIR spectrometer. The dispersions were diluted to give the absorption intensity lower than 1 .

\section{Tumor-sphere culture}

A single cell suspension was prepared using enzymatic (1x Trypsin-EDTA, Sigma Aldrich, \#T3924), and manual disaggregation (25 gauge needle) to create a single cell suspension. Cells were plated at a density of 500 cells $/ \mathrm{cm}^{2}$ in mammosphere medium (DMEM-F12/ B27/20ng/ml EGF/PenStrep) in non-adherent conditions, in culture dishes coated with (2-hydroxyethylmethacrylate) (poly-HEMA, Sigma, \#P3932) [12]. Cells were grown for 5 days and maintained in a humidified incubator at $37^{\circ} \mathrm{C}$ at an atmospheric pressure in 5\% (v/v) carbon dioxide/ air. After 5 days for culture, spheres $>50 \mu$ ? were counted using an eye graticule, and the percentage of tumorsphere formation was normalized to $100 \%$ for vehicle alone control ( $1=100 \%$ TSF) [12]. All experiments were performed in triplicate, three times independently, such that each data point represents the average of 9 replicates.

\section{Evaluation of CSC signalling pathways}

The Cignal Lenti reporter assay (luc) system (Qiagen) was chosen for monitoring the activity of several signal transduction pathways in MCF7 cells. The responsive luciferase constructs encode the firefly luciferase reporter gene under the control of a minimal (m) CMV promoter and tandem repeats of response elements for each pathway. The following constructs were used: $\mathrm{TCF} / \mathrm{LEF}$ (luc) for Wnt signal transduction (CLS-018L); STAT3(luc) for transcriptional activity of STAT3 (CLS6028L); RBP-Jk(luc) for Notch-induced signaling (CLS014L); ARE(luc) for Nrf2- and Nrf1-mediated antioxidant response (CLS-2020L); GAS(luc) for Interferon gamma-induced Stat1-signal transduction (CLS-009L); SMAD(luc) for TGF $\beta$-induced signal transduction (CLS017L). Briefly, 1 x $10^{5}$ MCF7-GFP cells were seeded in 12 -well plates. Once cells were attached, the viral particles were diluted 1:10 in complete culture media containing polybrene (sc-134220, Santa Cruz), and added to the cells. Puromycin treatment (P9620, Sigma) was started 48 hours later in order to select stably infected cells.

\section{Luciferase assays}

The Luciferase Assay System (E1501, Promega Kit) was used on all luciferase reporter MCF7 cells treated with GO. Briefly, $6 \times 10^{3}$ MCF7-GFP cells were seeded in black-walled 96-well plates and then were treated with GO $(50 \mu \mathrm{g} / \mathrm{ml})$. As controls, vehicle-treated cells were run in parallel. Four replicates were used for each condition. After 48 hours of treatment, luciferase assays were performed according to the manufacturer's instructions. Light signal was acquired for 2 minutes in photons/second in the Xenogen VivoVision IVIS Lumina (Caliper Life 
Sciences), and the results were analysed using Living Image 3.2 sofware (Caliper Life Sciences). Luminescence was normalized using SRB (to determine total cellular protein), as a measure of MCF7 cell viability.

\section{Anoikis and CSC differentiation analysis}

Following GO treatment, the CSC population was enriched by seeding on low-attachment plates. Under these conditions, the non-CSCs undergo anoikis (a form of apoptosis induced by lack of proper attachment) and CSCs are believed to survive. The expression of differentiation markers by the surviving "CSC fraction" was analyzed by FACS analysis. Briefly, $1 \times 10^{4} \mathrm{MCF} 7$ cells were treated with GO $(50 \mu \mathrm{g} / \mathrm{ml})$ for $48 \mathrm{~h}$ in 6-well plates, grown as a monolayer. Then, the monolayer cells were trypsinized and seeded in low-attachment plates in mammosphere media. After 10h under low-attachment conditions, MCF7 cells were spun down and incubated with CD24 (IOTest CD24PE, Beckman Coulter) and CD44 (APC mouse AntiHuman CD44, BD Pharmingen cat.559942) antibodies for 15 minutes on ice. Cells were rinsed twice and incubated with LIVE/DEAD dye (Fixable Dead Violet reactive dye; Invitrogen) for 10 minutes. Samples were then analyzed by FACS (Fortessa, BD Bioscence). Only the live population, as identified by the LIVE/DEAD dye staining, was analyzed for CD24/CD44 expression. Data were analysed using FlowJo software. Virtually identical results were also obtained using 7-AAD (7-Aminoactinomycin D; Life Technologies) to distinguish between the live and dead populations of cells (cell viability), during anoikis.

\section{ACKNOWLEDGEMENTS}

We thank the University of Manchester for providing start-up funds that contributed to the success of this study (to Federica Sotgia and Michael P. Lisanti). Aravind Vijayaraghavan, Maria Iliut and Andrea F. Verre were supported by Engineering and Physical Sciences Research Council (EPSRC) grants EP/G03737X/1 and $\mathrm{EP} / \mathrm{G} 035954 / 1$.

\section{REFERENCES}

1. Sinha N, Mukhopadhyay S, Das DN, Panda PK and Bhutia SK. Relevance of cancer initiating/stem cells in carcinogenesis and therapy resistance in oral cancer. Oral oncology. 2013; 49(9):854-862.

2. Xin H, Kong Y, Jiang X, Wang K, Qin X, Miao ZH, Zhu $Y$ and Tan W. Multi-drug-resistant cells enriched from chronic myeloid leukemia cells by Doxorubicin possess tumor-initiating-cell properties. Journal of pharmacological sciences. 2013; 122(4):299-304.

3. Easwaran H, Tsai HC and Baylin SB. Cancer epigenetics: tumor heterogeneity, plasticity of stem-like states, and drug resistance. Molecular cell. 2014; 54(5):716-727.

4. Dawood S, Austin L and Cristofanilli M. Cancer Stem Cells: Implications for Cancer Therapy. Oncology. 2014; 28(12).

5. Colak S and Medema JP. Cancer stem cells--important players in tumor therapy resistance. The FEBS journal. 2014; 281(21):4779-4791.

6. Filipova A, Seifrtova M, Mokry J, Dvorak J, Rezacova M, Filip S and Diaz-Garcia D. Breast cancer and cancer stem cells: a mini-review. Tumori. 2014; 100(4):363-369.

7. Scopelliti A, Cammareri P, Catalano V, Saladino V, Todaro $\mathrm{M}$ and Stassi G. Therapeutic implications of Cancer Initiating Cells. Expert opinion on biological therapy. 2009; 9(8):1005-1016.

8. Dean M. ABC transporters, drug resistance, and cancer stem cells. Journal of mammary gland biology and neoplasia. 2009; 14(1):3-9.

9. Reya T, Morrison SJ, Clarke MF and Weissman IL. Stem cells, cancer, and cancer stem cells. Nature. 2001; 414(6859):105-111.

10. Karsten U and Goletz S. What makes cancer stem cell markers different? SpringerPlus. 2013; 2(1):301.

11. Magee JA, Piskounova E and Morrison SJ. Cancer stem cells: impact, heterogeneity, and uncertainty. Cancer cell. 2012; 21(3):283-296.

12. Shaw FL, Harrison H, Spence K, Ablett MP, Simoes BM, Farnie $\mathrm{G}$ and Clarke RB. A detailed mammosphere assay protocol for the quantification of breast stem cell activity. Journal of mammary gland biology and neoplasia. 2012; 17(2):111-117.

13. Fillmore CM and Kuperwasser C. Human breast cancer cell lines contain stem-like cells that self-renew, give rise to phenotypically diverse progeny and survive chemotherapy. Breast cancer research : BCR. 2008; 10(2):R25.

14. Cojoc M, Mabert K, Muders MH and Dubrovska A. A role for cancer stem cells in therapy resistance: Cellular and molecular mechanisms. Seminars in cancer biology. 2014.

15. Skvortsov S, Debbage P, Lukas P and Skvortsova I. Crosstalk between DNA repair and cancer stem cell (CSC) associated intracellular pathways. Seminars in cancer biology. 2014.

16. Liu Z, Robinson JT, Sun X and Dai H. PEGylated nanographene oxide for delivery of water-insoluble cancer drugs. Journal of the American Chemical Society. 2008; 130(33):10876-10877.

17. Hernandez Y, Lotya M, Rickard D, Bergin SD and Coleman $J \mathrm{~N}$. Measurement of Multicomponent Solubility Parameters for Graphene Facilitates Solvent Discovery. Langmuir. 2010; 26(5):3208-3213.

18. Dreyer DR, Park S, Bielawski CW and Ruoff RS. The chemistry of graphene oxide. Chem Soc Rev. 2010; 39(1):228-240.

19. Liao KH, Lin YS, Macosko CW and Haynes CL. Cytotoxicity of graphene oxide and graphene in human 
erythrocytes and skin fibroblasts. ACS applied materials \& interfaces. 2011; 3(7):2607-2615.

20. Ruiz ON, Fernando KA, Wang B, Brown NA, Luo PG, McNamara ND, Vangsness M, Sun YP and Bunker CE. Graphene oxide: a nonspecific enhancer of cellular growth. ACS nano. 2011; 5(10):8100-8107.

21. Angeloni V, Tiberio P, Appierto V and Daidone MG. Implications of stemness-related signaling pathways in breast cancer response to therapy. Seminars in cancer biology. 2014.

22. Li D, Masiero M, Banham AH and Harris AL. The notch ligand JAGGED1 as a target for anti-tumor therapy. Frontiers in oncology. 2014; 4:254.

23. Yu H, Lee H, Herrmann A, Buettner $\mathrm{R}$ and Jove R. Revisiting STAT3 signalling in cancer: new and unexpected biological functions. Nature reviews Cancer. 2014; 14(11):736-746.

24. Lamb R, Ozsvari B, Lisanti CL, Tanowitz HB, Howell A, Martinez-Outschoorn UE, Sotgia F and Lisanti MP. Antibiotics that target mitochondria effectively eradicate cancer stem cells, across multiple tumor types: Treating cancer like an infectious disease. Oncotarget. 2015.

25. Zhou T, Zhang B, Wei P, Du Y, Zhou H, Yu M, Yan L, Zhang W, Nie G, Chen C, Tu Y and Wei T. Energy metabolism analysis reveals the mechanism of inhibition of breast cancer cell metastasis by PEG-modified graphene oxide nanosheets. Biomaterials. 2014; 35(37):9833-9843.

26. Chen GY, Chen CL, Tuan HY, Yuan PX, Li KC, Yang HJ and $\mathrm{Hu}$ YC. Graphene oxide triggers toll-like receptors/ autophagy responses in vitro and inhibits tumor growth in vivo. Advanced healthcare materials. 2014; 3(9):1486-1495.

27. Zhou H, Zhang B, Zheng J, Yu M, Zhou T, Zhao K, Jia Y, Gao X, Chen $\mathrm{C}$ and Wei T. The inhibition of migration and invasion of cancer cells by graphene via the impairment of mitochondrial respiration. Biomaterials. 2014; 35(5):15971607.

28. Chen GY, Meng CL, Lin KC, Tuan HY, Yang HJ, Chen $\mathrm{CL}$, Li KC, Chiang CS and Hu YC. Graphene oxide as a chemosensitizer: diverted autophagic flux, enhanced nuclear import, elevated necrosis and improved antitumor effects. Biomaterials. 2015; 40:12-22.

29. Bressan E, Ferroni L, Gardin C, Sbricoli L, Gobbato L, Ludovichetti F, Tocco I, Carraro A, Piattelli A and Zavan B. Graphene based scaffolds effects on stem cells commitment. Journal of translational medicine. 2014; 12(1):296.

30. $\mathrm{Ku} \mathrm{SH}$ and Park CB. Myoblast differentiation on graphene oxide. Biomaterials. 2013; 34(8):2017-2023.

31. Lee WC, Lim CH, Shi H, Tang LA, Wang Y, Lim CT and Loh KP. Origin of enhanced stem cell growth and differentiation on graphene and graphene oxide. ACS nano. 2011; 5(9):7334-7341.

32. Yang D, Li T, Xu M, Gao F, Yang J, Yang Z and Le W. Graphene oxide promotes the differentiation of mouse embryonic stem cells to dopamine neurons. Nanomedicine.
2014; 9(16):2445-2455.

33. Yoon HH, Bhang SH, Kim T, Yu T, Hyeon T and Kim BS. Dual Roles of Graphene Oxide in Chondrogenic Differentiation of Adult Stem Cells: Cell-Adhesion Substrate and Growth Factor-Delivery Carrier. Adv Funct Mater. 2014; 24(41):6455-6464.

34. Hummers WS and Offeman RE. Preparation of Graphitic Oxide. Journal of the American Chemical Society. 1958; 80(6):1339-1339.

35. Marcano DC, Kosynkin DV, Berlin JM, Sinitskii A, Sun ZZ, Slesarev A, Alemany LB, Lu W and Tour JM. Improved Synthesis of Graphene Oxide. ACS nano. 2010; 4(8):4806-4814. 